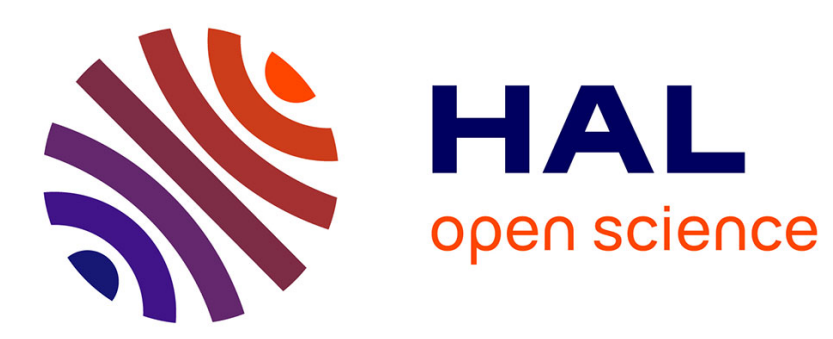

\title{
Traitement antihypertenseur prescrit aux patients avant leur première consultation dans un service spécialisé : comparaison entre 2001 et 2006
}

Olivier Steichen, Pierre-François Plouin

\section{- To cite this version:}

Olivier Steichen, Pierre-François Plouin. Traitement antihypertenseur prescrit aux patients avant leur première consultation dans un service spécialisé: comparaison entre 2001 et 2006. La Revue de Médecine Interne, 2008, 29 (10), pp.773-779. 10.1016/j.revmed.2008.01.002 hal-01171797

\section{HAL Id: hal-01171797 \\ https://hal.sorbonne-universite.fr/hal-01171797}

Submitted on 8 Jul 2015

HAL is a multi-disciplinary open access archive for the deposit and dissemination of scientific research documents, whether they are published or not. The documents may come from teaching and research institutions in France or abroad, or from public or private research centers.
L'archive ouverte pluridisciplinaire HAL, est destinée au dépôt et à la diffusion de documents scientifiques de niveau recherche, publiés ou non, émanant des établissements d'enseignement et de recherche français ou étrangers, des laboratoires publics ou privés. 


\title{
Traitement antihypertenseur prescrit aux patients avant leur première consultation dans un service spécialisé : comparaison entre 2001 et 2006
}

\section{Antihypertensive drugs prescribed to patients before their first consultation in a hypertension unit: comparison between 2001 and 2006}

O. Steichen ${ }^{\mathrm{a}, \mathrm{b}}$, P.-F. Plouin ${ }^{\mathrm{c}}$

${ }^{a}$ INSERM, U872, équipe 20 / laboratoire de santé publique et informatique médicale, 15 rue de l'école de médecine, 75006 Paris, France ; Université Pierre et Marie Curie - Paris6, UMR S 872, 75006 Paris, France ; Université Paris Descartes, UMR S 872, 75006 Paris, France.

${ }^{\mathrm{b}}$ AP-HP, hôpital Tenon, service de médecine interne, 4 rue de la Chine, Paris, F-75020 France ; Université Pierre et Marie Curie - Paris6, Faculté de Médecine, 75006 Paris, France.

${ }^{\mathrm{c}}$ AP-HP, Hôpital Européen Georges Pompidou, unité d'hypertension artérielle, 20 rue Leblanc, 75908 Paris cedex 15, France ; Université Paris Descartes, Faculté de Médecine, 75006 Paris, France.

\author{
Auteur correspondant : \\ Olivier Steichen \\ Service de Médecine Interne \\ 4, rue de la Chine \\ 75020 PARIS, France. \\ tel. $(+33 / 0) 156016815$ \\ fax $(+33 / 0) 156017082$ \\ mél. olivier.steichen@tnn.aphp.fr
}




\section{Résumé}

Propos. - Étudier l'évolution de la prescription des antihypertenseurs par des médecins nonspécialistes, au regard des bonnes pratiques établies par la recherche clinique.

Méthodes. - Comparaison des traitements prescrits aux patients avant leur première consultation dans un service d'hypertension artérielle en 2001 et 2006, avec une attention particulière pour les diurétiques thiazidiques dans les associations antihypertensives et les inhibiteurs de l'enzyme de conversion (IEC) chez les hypertendus à haut risque cardiovasculaire (diabète ou prévention secondaire).

Résultats. - La population comportait 1072 hypertendus de 53,9 ans d'âge moyen en 2001 et 1040 hypertendus de 55,6 ans d'âge moyen en 2006, avec une représentation égale des deux sexes. Les patients sous traitement antihypertenseur recevaient en moyenne 2,24 classes thérapeutiques en 2001 et 2,44 en $2006(\mathrm{p}=0,002)$. Trois classes ont progressé entre 2001 et 2006 : les inhibiteurs calciques étaient compris dans 49\% des traitements antihypertenseurs en 2001 et $56 \%$ en 2006 (p = 0,007), les antagonistes de récepteurs de l'angiotensine dans 28 et $42 \%$ ( $\mathrm{p}<0,001$ ) et les thiazidiques dans 31 et $39 \%$ ( $\mathrm{p}=0,001)$. En 2006, 55\% des associations antihypertensives comportaient un thiazidique contre $48 \%$ en $2001(\mathrm{p}=0,02)$. Les IEC faisaient partie de $31 \%$ des traitements prescrits aux patients à haut risque cardiovasculaire, de façon stable.

Conclusion. - Malgré une majoration de l'intensité des traitements, les thiazidiques restent sous utilisés dans les associations antihypertensives. Les IEC ne progressent pas chez les patients à haut risque cardiovasculaire, en dépit des données probantes acquises en leur faveur.

Mots clés

Adhésion à directive ; guides de bonne pratique ; hypertension artérielle ; antihypertenseurs 


\section{Abstract}

Purpose - To determine if trends in antihypertensive drug prescriptions by non-specialist physicians reflect evidence from clinical research.

Methods - Comparison of antihypertensive drugs prescribed to patients before they attended a hypertension clinic in 2001 and 2006, with a special consideration for thiazide diuretics in drug combinations and angiotensin converting enzyme inhibitors (ACEI) in hypertensive patients at high cardiovascular risk (diabetes or secondary prevention).

Results - Overall, 1072 hypertensive patients attended the hypertension clinic in 2001 (mean age 53.9 years) and 1040 in 2006 (mean age 55.6 years); both genders were equally represented. Patients already treated when they came at the consultation received a mean number of 2.24 antihypertensive drug classes in 2001 and 2.44 in $2006(\mathrm{p}=0.002)$. The prescription of three antihypertensive drugs increased between 2001 and 2006: Calcium channel blockers from $49 \%$ of treated patients in 2001 to $56 \%$ in 2006 ( $\mathrm{p}=0.007$ ), angiotensin receptor antagonists from 28 to $42 \%(\mathrm{p}<0.001)$ and thiazide diuretics from 31 to $39 \%(\mathrm{p}=0.001)$. Thiazide diuretics were included in $48 \%$ of the antihypertensive combinations in 2001 and $55 \%$ in $2006(p=0.02)$. The prescription of ACEI in high-risk patients remained stable around $31 \%$.

Conclusion - Antihypertensive treatments were more intensive in 2006 than 2001, but thiazide diuretics remained underused in drug combinations. The prescription of ACEI did not increase in patients at high cardiovascular risk despite convincing evidence of their benefit.

Keywords

Guideline adherence; practice guidelines; hypertension; antihypertensive agents 


\section{Introduction}

L'hypertension artérielle (HTA) est une maladie fréquente, contribuant de façon significative à la morbi-mortalité cardiovasculaire et rénale. Ses conséquences peuvent être limitées par une prise en charge structurée, centrée par des interventions hygiéno-diététiques et pharmacologiques visant à réduire le niveau de la pression artérielle et des autres facteurs de risque cardiovasculaires.

De nombreuses études cliniques ont été entreprises dans ce domaine et leurs résultats permettent de rationaliser la prise en charge des malades hypertendus. Des sociétés savantes et des institutions nationales ou internationales ont édité des guides pour la pratique clinique dans le domaine de l'hypertension, à l'intention des médecins non-spécialistes [1-5].

Néanmoins, une discordance importante persiste entre les pratiques recommandées et les pratiques observées [6]. La plupart des études d'évaluation des pratiques dans le domaine de l'hypertension souffrent de faiblesses méthodologiques qui limitent leur interprétation :

- absence d'indicateur explicite de bonne pratique thérapeutique,

- absence de considération pour la comorbidité des patients,

- absence d'évaluation de l'impact des pratiques non conformes sur le contrôle de la pression artérielle, et a fortiori sur la morbi-mortalité cardiovasculaire.

En tentant nous affranchir de ces défauts, nous décrivons ici l'évolution du traitement antihypertenseur prescrit par des médecins non-spécialistes en HTA à des patients qu'ils ont adressés à une consultation spécialisée en 2001 et 2006. L'analyse est menée à la lumière du consensus admis en France à ces deux époques, reflété les recommandations pour la prise en charge de l'HTA publiées par l'ANAES (Agence Nationale d'Accréditation et d'Évaluation en Santé) en 2000 [1] et actualisées par la HAS (Haute Autorité de Santé) en 2005 [2].

\section{Méthodes}

\section{Patients}

Tous les patients hypertendus consultant pour la première fois en 2001 ou 2006 dans le service d'hypertension artérielle de l'hôpital européen Georges Pompidou ont été inclus. Les patients dont l'HTA n'était pas confirmée (pression artérielle normale en l'absence de traitement antihypertenseur) et ceux dont la première visite dans le service avait directement lieu en hôpital de jour ou de semaine ont été exclus.

\section{Données}

L'observation médicale utilisée dans le service est informatisée depuis 1975 ; les données cliniques et paracliniques des patients sont enregistrées dans une base de donnée hospitalière [7]. Il est possible d'accéder par des requêtes informatiques à l'histoire médicale, au traitement en cours lors de la visite, à l'évaluation du contrôle de l'HTA et des cofacteurs de risque cardiovasculaire, au résultat de l'enquête étiologique et au retentissement sur les organes cibles.

Les caractéristiques suivantes des patients au moment de la première consultation ont été extraites : âge, sexe, indice de masse corporelle (poids/taille ${ }^{2}$ ), durée d'évolution de l'hypertension, traitement en cours [dont score thérapeutique $=$ nombre de classes pharmacologiques antihypertensives différentes prescrites au même malade], pressions artérielles systolique (PAS) et diastolique (PAD) [moyenne de 3 mesures automatiques par moniteur], existence d'un tabagisme actuel et/ou passé, antécédent connu de dyslipidémie, de diabète, d'insuffisance rénale, d'accident vasculaire cérébral (AVC), d'artériopathie oblitérante des membres inférieurs (AOMI), de cardiopathie ischémique, d'insuffisance cardiaque. 


\section{Indicateurs}

Les indicateurs préférés pour évaluer l'adéquation de la prise en charge pharmacologique des hypertendus sont relatifs (1) à la place donnée aux diurétiques thiazidiques et (2) au choix de classes thérapeutiques adaptées à la comorbidité du patient [8]. Les recommandations françaises placent les diurétiques thiazidiques au plus tard en seconde ligne thérapeutique en 2000 et au plus tard en troisième ligne en 2005. Nous avons donc retenu comme premier indicateur thérapeutique la place des diurétiques thiazidiques dans les associations antihypertensives. Le niveau de pression artérielle des malades sous association antihypertensive a été comparé en fonction de la présence ou non d'un diurétique thiazidique, c'est-à-dire de la conformité ou non du traitement aux recommandations.

Les patients à haut risque cardiovasculaire représentent un sous-groupe important des hypertendus, au sein duquel l'évolution des pratiques peut être analysée avec une bonne puissance statistique. L'intérêt des IEC à fortes doses - $10 \mathrm{mg}$ pour le ramipril, $8 \mathrm{mg}$ pour le perindopril, $4 \mathrm{mg}$ pour le trandolapril $[9,10]$ — est reconnu chez ces patients de façon consensuelle [11,12]. Nous avons étudié la prescription des IEC chez les patients à haut risque cardiovasculaire, définis selon les critères d'inclusion de l'étude HOPE [13] : antécédent personnel de cardiopathie ischémique, d'AVC, d'AOMI ou de diabète (tous les patients de notre étude souffrent d'une HTA comme facteur de risque ajouté au diabète). Dans la mesure où la molécule et la dose ne sont pas saisies dans des champs spécifiques de l'observation informatisée, toutes les prescriptions d'IEC ont été considérées de façon indistincte dans notre étude.

\section{Statistiques}

Les données des patients adressés en consultation en 2001 et en 2006 sont présentées en nombre absolu (pourcentage) pour les variables binaires et en moyenne ( \pm écart-type) pour les variables quantitatives. Pour chaque variable, le nombre de patients avec des données disponibles est également indiqué. Les valeurs de 2001 et 2006 sont comparées par le test du Chi-2 (variables binaires) et le test-t de Student (variables quantitatives, après vérification graphique de la normalité approximative de la distribution), avec un seuil de significativité à 0,05. En cas de différence significative, des comparaisons ajustées par régression logistique ou linéaire ont été entreprises pour contrôler l'effet des facteurs de confusion potentiels. L'influence de la comorbidité (diabète, complication de l'athérosclérose, insuffisance cardiaque, insuffisance rénale) sur la nature des classes antihypertensives prescrites a été étudiée. Les analyses ont été réalisées à l'aide du logiciel Stata 8.2 (StataCorp, College Station, Texas, États-Unis).

\section{Résultats}

La table 1 résume les caractéristiques des patients consultant pour la première fois dans le service d'HTA de l'hôpital européen Georges Pompidou en 2001 et 2006. Les patients sont plus âgés en 2006. Ils ont également une HTA plus ancienne et plus fréquemment une insuffisance cardiaque. Enfin, ils sont traités plus intensivement (pourcentage de patients traités, score thérapeutique des patients traités) et leur pression artérielle est mieux contrôlée (niveau de PAS et de PAD, pourcentage de patients contrôlés). Ces différences d'intensité thérapeutique et de contrôle de l'HTA restent du même ordre de grandeur et statistiquement significatives après ajustement sur les facteurs de confusion potentiels (dont l'âge et l'insuffisance cardiaque ; résultats numériques non présentés).

La table 2 révèle que l'incrément thérapeutique observé en 2006 se fait au profit de 3 classes d'antihypertenseurs : les inhibiteurs calciques, les antagonistes de l'angiotensine 2 (ARA2) et les diurétiques thiazidiques. La table 3 montre l'évolution de la prescription de ces trois classes thérapeutiques dans les sous-groupes définis par la comorbidité. Les inhibiteurs calciques sont déjà très prescrits en 2001 aux diabétiques, aux insuffisants cardiaques et aux patients ayant une 
complication de l'athérosclérose ; ils progressent essentiellement chez les patients sans comorbidité. Au contraire, la progression des thiazidiques est surtout sensible chez les malades ayant une comorbidité significative. Les antagonistes de l'angiotensine 2 sont utilisés de façon croissante dans tous les sous-groupes.

La table 2 montre aussi que le choix des antihypertenseurs prescrits en monothérapie est relativement stable entre 2001 et 2006, avec une très faible représentation des diurétiques thiazidiques.

Concernant les associations antihypertensives, les diurétiques thiazidiques en font partie dans $48 \%$ des cas (228/476) en 2001 et 55\% (305/552) en $2006(\mathrm{p}=0,02)$. Cependant, malgré les recommandations, ils demeurent employés dans moins de deux tiers des trithérapies : 55\% (75/137) en 2001 et $62 \%(119 / 191)$ en 2006. Ces résultats ne sont pas modifiés par l'exclusion des malades souffrant d'insuffisance cardiaque ou rénale, chez qui les diurétiques de l'anse peuvent être préférés aux thiazidiques (résultats numériques non présentés). Les thiazidiques stagnent à $35 \%$ dans les bithérapies en 2001 (73/209) et 2006 (70/199). Les patients sous bithérapie, et dans une moindre mesure sous trithérapie, ont pourtant une hypertension significativement mieux contrôlée lorsque leur traitement comporte un thiazidique (table 4).

La prescription des IEC chez les patients à haut risque cardiovasculaire ne progresse pas : $34 \%$ $(68 / 201)$ en 2001 et 29\% (71/243) en $2006(\mathrm{p}=0,30)$. Cependant, la proportion des patients à haut risque recevant un traitement inhibiteur du système rénine angiotensine augmente de $59 \%$ $(118 / 201)$ en 2001 à $71 \%(172 / 243, p=0,008)$ en 2006, grâce à la progression des ARA2 de $28 \%$ $(56 / 201)$ à $46 \%(111 / 243, \mathrm{p}<0,001)$.

\section{Récapitulatif}

1. La prise en charge de l'hypertension des patients adressés en consultation est globalement plus intensive en 2006 qu'en 2001.

2. Les classes thérapeutiques mises à contribution pour cette intensification thérapeutique sont les inhibiteurs calciques, les ARA2 et les diurétiques thiazidiques.

3. Cependant, les diurétiques thiazidiques restent sous-utilisés et sont en particulier absents de $2 / 3$ des bithérapies et de $1 / 3$ des trithérapies en 2006. Pourtant, l'hypertension est mieux contrôlée avec une bithérapie ou une trithérapie qui comporte un thiazidique.

4. La prescription des IEC ne progresse pas chez les patients à haut risque cardiovasculaire, alors que celle des ARA2 augmente fortement.

\section{Discussion}

\section{Limites de l'étude}

Ces résultats ont été établis sur un effectif important de patients dont les caractéristiques cliniques sont connues et prises en compte dans les analyses. L'effet des facteurs de confusion potentiels et les spécificités des sous-groupes pertinents ont été étudiés. Toutefois, certaines limites méthodologiques doivent être soulignées avant de commenter les résultats.

Premièrement, les malades de notre étude et les médecins référents sont sélectionnés ; il n'est pas garanti que les résultats obtenus reflètent les habitudes de prescription des antihypertenseurs en général. En effet, les patients inclus sont suffisamment difficiles à prendre en charge pour être adressés en consultation d'hypertension. Il est donc envisageable que les choix thérapeutiques soient plus complexes que dans le cas général et se fassent plus fréquemment hors recommandations. D'autre part, pour qu'un médecin adresse son patient en consultation d'hypertension, il doit avoir identifié l'intérêt pour le patient d'une prise en charge spécialisée. Or, les médecins qui 
reconnaissent le mieux leurs limites dans la prise en charge de l'HTA sont également ceux qui se conforment le plus aux recommandations [14]. Le cas échéant, ce biais de sélection devrait donc plutôt induire une surestimation de la proportion des pratiques conformes aux recommandations.

Deuxièmement, nos résultats sont sujets aux limites des études transversales, qui ne peuvent pas établir de relations de causalité, en raison du mauvais contrôle des facteurs de confusion et de l'absence de donnée sur la chronologie des événements. En particulier, le lien de causalité entre l'absence de thiazidique dans une association médicamenteuse et le moins bon contrôle de l'hypertension est impossible à prouver, même si la bonne synergie des thiazidiques avec les autres classes thérapeutiques le rend plausible.

Troisièmement, nos résultats sont sujets aux limites des études rétrospectives concernant la fiabilité et la complétude du recueil des données. Néanmoins, la saisie a été effectuée de façon prospective durant les consultations et la proportion de données manquantes est toujours inférieure à $20 \%$. La seule exception notable est l'artériopathie oblitérante des membres inférieurs en 2001. En effet, une question spécifique sur l'abolition des pouls périphériques a été ajoutée à l'observation informatisée dans le courant de l'année 2001. Le nombre de données manquantes sur la présence d'une AOMI avant l'introduction de cette question est important. L'AOMI étant un des critères de haut risque cardiovasculaire, les données manquantes pourraient conduire à une sous-estimation de la prévalence des patients à haut risque en 2001. Pour prévenir ce biais, nous avons imputé les données manquantes d'AOMI en 2001 jusqu'à atteindre le même niveau d'information qu'en 2006. En effet, après simulation des données manquantes par une régression logistique multivariée prenant en compte les facteurs de risque cardiovasculaire classiques (age, sexe, niveau de pression artérielle, dyslipidémie, diabète, tabagisme), il est possible d'avoir une estimation non biaisée de la prévalence des patients à haut risque cardiovasculaire [15].

Toujours au titre des limites du recueil rétrospectif des données, certaines informations souhaitables pour l'interprétation des résultats n'étaient pas disponibles à travers une requête informatique. Ainsi, l'histoire thérapeutique des patients avant leur arrivée en consultation n'est pas connue, alors que l'hypertension est traitée depuis $71 / 2$ ans en moyenne. La qualification des médecins référents n'est pas non plus accessible. À ce sujet, une étude antérieure dans la même structure de soins trouvait $60 \%$ des patients adressés par un généraliste, 20\% par un autre spécialiste (neurologue, cardiologue, obstétricien, etc.), $10 \%$ par un médecin du travail et 10\% de malades consultant spontanément [7]. En outre, les patients consultant spontanément sont probablement moins traités que les patients adressés par leur médecin. Une modification de leur proportion pourrait donc biaiser la comparaison du pourcentage de patients sous traitement à leur arrivée en consultation et, dans une moindre mesure, la comparaison du score thérapeutique des patients traités.

\section{Comparaison à d'autres études}

Nos résultats sont à mettre en rapport avec l'évolution globale de la prescription des traitements antihypertenseurs en France métropolitaine durant la même période, selon la Caisse Nationale d'Assurance Maladie (CNAM) [16]. Ces données montrent que chez les patients traités par antihypertenseurs, les IEC régressent de 31 à 29\% entre 2001 et 2006, alors que les béta-bloquants sont stables à 39\% et les inhibiteurs calciques à 29\%. Les deux seules classes antihypertensives qui progressent sont les ARA2 (de 23 à 37\%) et les thiazidiques (de 38 à 41\%). Ces deux classes supportent donc à elles seules l'intensification des traitements dont témoigne la progression des associations thérapeutiques : les bithérapies passent de 33 à 35\%, les trithérapies de 16 à $18 \%$ et les quadrithérapies ou plus passent de 7 à $8 \%$. Ces grandes tendances corroborent la réalité de l'intensification thérapeutique observée dans notre population, ainsi que la progression spectaculaire des ARA2.

Nous n'avons pas trouvé d'autre évaluation des prescriptions antihypertensives dans une population comparable à la notre — avec une représentation importante des hypertensions secondaires, 
résistantes ou compliquées - et durant la même période. Une étude américaine s'est intéressée à l'évolution entre 2001 et 2004 des prescriptions faites par un panel de médecins représentatifs pour l'ensemble des États-Unis, exerçant en cabinet pour la plupart [17]. L'intensité du traitement dans cette population d'hypertendus non sélectionnés est globalement moindre que dans notre étude. Les thiazidiques et les ARA2 progressent de façon significative (respectivement de 20 à $25 \%$ et de 15 à $25 \%$ ). Les autres classes restent relativement stables : IEC autour de 35\%, inhibiteurs calciques autour de $25 \%$ et béta-bloquants autour de $20 \%$. Les tendances évolutives sont donc les mêmes que celles que nous observons dans notre population, hormis le succès moins marqué des inhibiteurs calciques, et sont exactement les mêmes que celles qui sont observées dans la population générale en France. L'influence de la comorbidité et l'effet des facteurs de confusion potentiels n'ont pas été étudiés dans ce travail.

\section{Interprétation}

Par rapport à leurs homologues américains ou britanniques, les médecins français ont peu l'habitude de prescrire des thiazidiques [18], ce dont témoigne leur très faible utilisation en monothérapie. Il faut noter en outre que les guides de bonne pratique français ont rétrogradé dans la promotion des thiazidiques entre 2001 (recommandés au plus tard en deuxième position) et 2005 (recommandés au plus tard en troisième position). Dans ce contexte, la généralisation des associations fixes constitue probablement le principal facteur de progression des thiazidiques.

Deux tiers des patients à haut risque cardiovasculaire ne reçoivent toujours pas d'IEC en 2006, sans aucun progrès noté depuis 2001. De plus, il est fort probable qu'une proportion non négligeable des molécules ou des doses prescrites ne soient pas celles qui ont fait leur preuve dans cette indication. La sous prescription des IEC chez les patients à haut risque peut s'expliquer par un manque d'information ou de conviction des médecins. En effet, l'intérêt spécifique des IEC chez les patients à haut risque cardiovasculaire est évoqué dans l'argumentaire des recommandations françaises de 2005, mais le niveau de preuve est jugé insuffisant pour en faire une recommandation thérapeutique à part entière figurant dans le document de synthèse diffusé aux généralistes. Pourtant, les sociétés européenne et américaine de cardiologie attribuent le plus haut grade à la recommandation des IEC chez les patients à haut risque cardiovasculaire [11,12]. Même connaissant et admettant l'intérêt des IEC chez ces patients, il est possible que les prescripteurs aient jugé adéquat de prescrire un ARA2 à la place, par analogie avec leur équivalence dans l'insuffisance cardiaque et les néphropathies chroniques. Or, les données actuelles les plus probantes sont en faveur de la supériorité des IEC sur les ARA2 chez les malades à haut risque cardiovasculaire [19].

Les ARA2 devraient donc être prescrits aux patients à haut risque cardiovasculaire seulement en cas d'intolérance aux IEC, en particulier en lors d'une toux sous IEC sans autre explication. Cette complication touche 5 à $20 \%$ des patients traités [20]. Elle ne peut donc pas expliquer à elle seule la stagnation des IEC observée dans notre étude et à l'échelon national par la CNAM. La forte promotion des nouvelles classes thérapeutiques, dont les ARA2, par les laboratoires pharmaceutiques contribue probablement à leur succès [18,21]. Cependant, l'amplitude du phénomène est considérable en France puisque la prescription des ARA2 y dépasse celles des IEC, contrairement à ce qui est observé dans d'autres pays européens comme l'Allemagne, l'Espagne, l'Italie et l'Espagne, ou encore aux Etats-Unis [22]. Cette particularité semble marquer une propension des prescripteurs français à favoriser les innovations thérapeutiques, au-delà de ce que les données acquises par la recherche clinique permettent de justifier.

\section{Perspectives}

Nos résultats plaident pour une promotion plus engagée des thiazidiques dans les associations antihypertensives et des IEC chez les patients à haut risque vasculaire. Compte tenu des données accumulées en faveur de ces traitements, il est souhaitable qu'ils soient plus explicitement 
recommandés par les guides de bonne pratique français.

Par ailleurs, nous prévoyons d'évaluer la valeur ajoutée de la prise en charge des hypertendus dans un service spécialisé. Cette évaluation se fera entre autres à l'aide d'un certain nombre d'indicateurs de conformité aux recommandations, en terme de prescription et de résultat thérapeutique. Une approche qualitative pour comprendre la justification des décisions hors recommandations sera associée.

\section{Conclusion}

Un progrès significatif peut être noté dans la prescription des antihypertenseurs par les médecins qui adressent des patients hypertendus à une consultation spécialisée. Ce progrès est surtout quantitatif, comme l'indique l'augmentation de l'intensité des traitements, et s'accompagne d'un meilleur contrôle de la pression artérielle. En revanche, on n'observe pas l'évolution qualitative suggérée par les essais récents de prévention cardiovasculaire. Les conclusions de ces essais auraient dû augmenter la fréquence de l'emploi des diurétiques en monothérapie et surtout dans les associations antihypertensives, de même que la fréquence de l'emploi des IEC chez les hypertendus à haut risque vasculaire. L'adoption de ces mesures devrait permettre un contrôle encore meilleur de l'hypertension et un bénéfice supplémentaire en terme de morbi-mortalité. Il est nécessaire que les enseignements de ces essais soient pris en compte de façon claire dans les recommandations, afin d'éviter une prescription biaisée par l'industrie pharmaceutique au profit des classes thérapeutiques plus récentes.

\section{Remerciements}

Ce travail a bénéficié d'une subvention de la Société Française d'Hypertension Artérielle (SFHTA).

\section{Conflits d'intérêt}

OS : aucun

PFP : aucun 


\section{Références}

[1] Agence Nationale d'Accréditation et d'Évaluation en Santé (ANAES). Prise en charge de l'hypertension artérielle essentielle chez l'adulte. Ann Endocrinol (Paris) 2000; 61:479-88.

[2] Haute Autorité de Santé (HAS). Prise en charge de l'hypertension artérielle essentielle chez l'adulte. actualisation 2005. J Mal Vasc 2006; 31:16-33.

[3] Chobanian AV, Bakris GL, Black HR, Cushman WC, Green LA, Izzo JLJ et al. Seventh report of the joint national committee on prevention, detection, evaluation, and treatment of high blood pressure. Hypertension 2003; 42:1206-52.

[4] European Society of Hypertension (ESH), European Society of Cardiology (ESC). 2003 guidelines for the management of arterial hypertension.. J Hypertens 2003; 21:1011-53.

[5] Whitworth JA. 2003 world health organization (WHO)/international society of hypertension (ISH) statement on management of hypertension. J Hypertens 2003; 21:1983-92.

[6] Milchak JL, Carter BL, James PA, Ardery G. Measuring adherence to practice guidelines for the management of hypertension: an evaluation of the literature. Hypertension 2004; 44:602-8.

[7] Degoulet P, Chatellier G, Devriès C, Lavril M, Ménard J. Computer-assisted techniques for evaluation and treatment of hypertensive patients. Am J Hypertens 1990; 3:156-63.

[8] Milchak JL, Carter BL, Ardery G, Black HR, Bakris GL, Jones DW et al. Development of explicit criteria to measure adherence to hypertension guidelines. J Hum Hypertens 2006; 20:42633.

[9] Dagenais GR, Pogue J, Fox K, Simoons ML, Yusuf S. Angiotensin-converting-enzyme inhibitors in stable vascular disease without left ventricular systolic dysfunction or heart failure: a combined analysis of three trials. Lancet 2006; 368:581-8.

[10] Danchin N, Cucherat M, Thuillez C, Durand E, Kadri Z, Steg PG. Angiotensin-converting enzyme inhibitors in patients with coronary artery disease and absence of heart failure or left ventricular systolic dysfunction: an overview of long-term randomized controlled trials. Arch Intern Med. 2006; 166:787-96.

[11] Lopez-Sendon J, Swedberg K, McMurray J, Tamargo J, Maggioni AP, Dargie H et al. Expert consensus document on angiotensin converting enzyme inhibitors in cardiovascular disease. The task force on ACE-inhibitors of the European Society of Cardiology. Eur Heart J 2004; 25:1454-70. [12] Smith SCJ, Allen J, Blair SN, Bonow RO, Brass LM, Fonarow GC et al. AHA/ACC guidelines for secondary prevention for patients with coronary and other atherosclerotic vascular disease: 2006 update: endorsed by the National Heart, Lung, and Blood Institute. Circulation 2006; 113:2363-72.

[13] Yusuf S, Sleight P, Pogue J, Bosch J, Davies R, Dagenais G. Effects of an angiotensinconverting-enzyme inhibitor, ramipril, on cardiovascular events in high-risk patients. the heart outcomes prevention evaluation study investigators. N Engl J Med 2000; 342:145-53.

[14] Steinman MA, Fischer MA, Shlipak MG, Bosworth HB, Oddone EZ, Hoffman BB et al. Clinician awareness of adherence to hypertension guidelines. Am J Med 2004; 117:747-54.

[15] Donders AR, van der Heijden GJ, Stijnen T, Moons KG. Review: a gentle introduction to imputation of missing values. J Clin Epidemiol 2006; 59:1087-91.

[16] Samsons S, Ricordeau P, Pepin S, Tilly B, Weill A, Alleman H. Hypertension artérielle et facteurs de risque associés : évolution des traitements entre 2000 et 2006. Points de Repère 2007; 10:1-8.

[17] Stafford RS, Monti V, Furberg CD, Ma J. Long-term and short-term changes in antihypertensive prescribing by office-based physicians in the united states. Hypertension 2006; 48:213-8.

[18] Fretheim A, Oxman AD. International variation in prescribing antihypertensive drugs: its extent and possible explanations. BMC Health Serv Res 2005; 5:21.

[19] The Blood Pressure Lowering Trialists' Collaboration, Turnbull F, Neal B, Pfeffer M, Kostis J, Algert C et al. Blood pressure-dependent and independent effects of agents that inhibit the reninangiotensin system. J Hypertens 2007; 25:951-8. 
[20] Israili ZH, Hall WD. Cough and angioneurotic edema associated with angiotensin-converting enzyme inhibitor therapy. A review of the literature and pathophysiology. Ann Intern Med 1992; 117:234-42.

[21] Wang TJ, Ausiello JC, Stafford RS. Trends in antihypertensive drug advertising, 1985-1996. Circulation 1999; 99:2055-7.

[22] Wang YR, Alexander GC, Stafford RS. Outpatient hypertension treatment, treatment intensification, and control in Western Europe and the United States. Arch Intern Med 2007; 167:141-7. 


\begin{tabular}{|c|c|c|c|c|c|}
\hline & \multicolumn{2}{|c|}{$2001(\mathrm{~N}=1072)$} & \multicolumn{2}{|c|}{$2006(\mathrm{~N}=1040)$} & \multirow[b]{2}{*}{$\mathrm{p}$} \\
\hline & $\begin{array}{c}\text { Données } \\
\text { disponibles }\end{array}$ & $\begin{array}{l}\text { Valeur de la } \\
\text { variable }\end{array}$ & $\begin{array}{c}\text { Données } \\
\text { disponibles }\end{array}$ & $\begin{array}{l}\text { Valeur de la } \\
\text { variable }\end{array}$ & \\
\hline Hommes & $1072(100 \%)$ & $525(49 \%)$ & $1040(100 \%)$ & $522(50 \%)$ & 0,56 \\
\hline Age (années) & $1072(100 \%)$ & $53,9( \pm 14,5)$ & $1040(100 \%)$ & $55,6( \pm 15,1)$ & 0,008 \\
\hline Indice de masse corporelle $\left(\mathrm{kg} / \mathrm{m}^{2}\right)$ & $1045(97 \%)$ & $27,1( \pm 5,1)$ & $1003(96 \%)$ & $27,4( \pm 5,24)$ & 0,24 \\
\hline Dyslipidémie connue & $1013(94 \%)$ & $355(35 \%)$ & $990(95 \%)$ & $352(36 \%)$ & 0,81 \\
\hline Diabète connu & $1017(95 \%)$ & $116(11 \%)$ & $989(95 \%)$ & $134(14 \%)$ & 0,15 \\
\hline Consommation de tabac dans la vie & $1021(95 \%)$ & $417(41 \%)$ & $997(96 \%)$ & $400(40 \%)$ & 0,74 \\
\hline Consommation de tabac en cours & $1009(94 \%)$ & $154(15 \%)$ & $996(96 \%)$ & $121(12 \%)$ & 0,04 \\
\hline Insuffisance rénale connue & $1007(94 \%)$ & $42(4 \%)$ & $983(95 \%)$ & $45(5 \%)$ & 0,66 \\
\hline Insuffisance cardiaque connue & $937(87 \%)$ & $38(4 \%)$ & $952(92 \%)$ & $84(9 \%)$ & $<0,001$ \\
\hline Insuffisance coronaire connue & $930(87 \%)$ & $44(5 \%)$ & $967(93 \%)$ & $56(6 \%)$ & 0,30 \\
\hline Artériopathie oblitérante & $350(33 \%)$ & $28(8 \%)$ & $879(85 \%)$ & $72(8 \%)$ & 0,91 \\
\hline Accident vasculaire cérébral & $838(78 \%)$ & $69(8 \%)$ & $831(80 \%)$ & $72(9 \%)$ & 0,75 \\
\hline Patients à haut risque cardiovasculaire* & $1067(100 \%)$ & $235(22 \%)$ & $1029(99 \%)$ & $260(25 \%)$ & 0,08 \\
\hline Durée d'évolution de l'HTA (années) & $1009(94 \%)$ & $8,3( \pm 9,7)$ & $969(93 \%)$ & $9,5( \pm 10,4)$ & 0,008 \\
\hline Patients traités & $980(91 \%)$ & $752(77 \%)$ & $969(93 \%)$ & $799(82 \%)$ & 0,002 \\
\hline Score thérapeutique ${ }^{* *}$ (pour les patients traités) & $752(100 \%)$ & $2,24( \pm 1,27)$ & $799(100 \%)$ & $2,44( \pm 1,32)$ & 0,002 \\
\hline Pression artérielle systolique $(\mathrm{mmHg})$ & $1058(99 \%)$ & $151,5( \pm 24,6)$ & $1032(99 \%)$ & $147,8( \pm 22,8)$ & $<0,001$ \\
\hline Pression artérielle diastolique $(\mathrm{mmHg})$ & $1058(99 \%)$ & $89,5( \pm 13,1)$ & $1032(99 \%)$ & $85,6( \pm 13,7)$ & $<0,001$ \\
\hline HTA contrôlée $(\leq 140 / 90 \mathrm{mmHg})$ & $1058(99 \%)$ & $324(31 \%)$ & $1032(99 \%)$ & $382(37 \%)$ & 0,002 \\
\hline
\end{tabular}

Table 1. Caractéristiques des patients adressés en consultation d'hypertension. Valeurs exprimées en moyenne (土écart-type) ou nombre de patients (pourcentage) selon les cas, respectivement comparées par test t de Student et test du Chi-2.

*Pour une estimation non biaisée de la prévalence du haut risque cardiovasculaire, des données d'AOMI ont été générées pour remplacer des données manquantes en 2001. L’imputation des données manquantes a été réalisée par régression logistique multivariée sur les facteurs de risque cardiovasculaires classiques (age, sexe, niveau de pression artérielle, dyslipidémie, diabète, tabagisme).

** Le score thérapeutique est le nombre de classes pharmacologiques antihypertensives différentes prescrites au même malade. 


\section{Tous patients sous traitement}

Nombre de patients

Inhibiteur calcique

Béta-bloquant

Central

Bloqueur du SRA*

Antagoniste de l'angiotensine 2

Inhibiteur de l'enzyme de conversion

Diurétique

Diurétique thiazidique

Diurétique de l'anse

Anti-aldostérone

Diurétique épargneur de potassium

Vasodilatateur

Alpha-bloquant

Vasodilatateur direct

\section{Patients sous monothérapie}

Nombre de patients

Inhibiteur calcique

Béta-bloquant

Antagoniste de l'angiotensine 2

Inhibiteur de l'enzyme de conversion

Central

Diurétique thiazidique

Anti-aldostérone

Autres diurétiques et vasodilatateurs
752

$369(49 \%)$

$309(41 \%)$

$127(17 \%)$

$396(53 \%)$

$211(28 \%)$

$196(26 \%)$

$346(46 \%)$

$234(31 \%)$

$78(10 \%)$

$57(8 \%)$

$35(5 \%)$

$60(8 \%)$

$54(7 \%)$

$6(1 \%)$

276

$89(32 \%)$

$69(25 \%)$

$43(16 \%)$

$36(13 \%)$

$14(5 \%)$

$6(2 \%)$

$11(4 \%)$

$8(3 \%)$
799

447 (56\%)

0,007

$326(41 \%)$

0,91

$129(16 \%)$

0,69

$497(62 \%)$

$<0,001$

$333(42 \%)$

$<0,001$

$186(23 \%)$

0,20

$416(52 \%)$

0,02

$315(39 \%)$

0,001

$70(9 \%)$

0,28

$63(8 \%)$

0,82

$29(4 \%)$

0,31

$48(6 \%)$

0,13

$35(4 \%)$

0,02

$14(2 \%)$

0,10

\section{7}

$94(38 \%)$

0,16

$56(23 \%)$

0,53

$43(17 \%)$

0,57

$28(11 \%)$

0,55

$10(4 \%)$

0,58

$10(4 \%)$

0,21

$4(2 \%)$

0,11

Table 2. Classes thérapeutiques prescrites aux patients à leur arrivée en consultation d'hypertension. Données exprimées en nombre de patients (pourcentage) et comparées par test du Chi-2.

*SRA = Système Rénine Angiotensine 


\begin{tabular}{|c|c|c|c|}
\hline & Valeur en 2001 & Valeur en 2006 & $\mathrm{p}$ \\
\hline \multicolumn{4}{|l|}{ Diabète } \\
\hline Inhibiteurs calciques & $59 / 97(61 \%)$ & $71 / 120(59 \%)$ & 0,80 \\
\hline Antagoniste de l'angiotensine 2 & $37 / 97(38 \%)$ & $67 / 120(56 \%)$ & 0,01 \\
\hline Thiazidiques & $33 / 97(34 \%)$ & $58120(48 \%)$ & 0,03 \\
\hline \multicolumn{4}{|l|}{ Insuffisance rénale } \\
\hline Inhibiteurs calciques & $21 / 36(58 \%)$ & $33 / 41(80 \%)$ & 0,03 \\
\hline Antagoniste de l'angiotensine 2 & $11 / 36(31 \%)$ & $20 / 41(49 \%)$ & 0,10 \\
\hline Thiazidiques & $6 / 36(17 \%)$ & $16 / 41(39 \%)$ & 0,03 \\
\hline \multicolumn{4}{|l|}{ Insuffisance cardiaque } \\
\hline Inhibiteurs calciques & $23 / 37(62 \%)$ & $43 / 72(60 \%)$ & 0,81 \\
\hline Antagoniste de l'angiotensine 2 & $13 / 37(35 \%)$ & $33 / 72(46 \%)$ & 0,28 \\
\hline Thiazidiques & $11 / 37(30 \%)$ & $32 / 72(44 \%)$ & 0,14 \\
\hline \multicolumn{4}{|l|}{ Complication de l'athérosclérose* } \\
\hline Inhibiteurs calciques & $76 / 120(63 \%)$ & $98 / 155(63 \%)$ & 0,99 \\
\hline Antagoniste de l'angiotensine 2 & $25 / 120(21 \%)$ & $66 / 155(43 \%)$ & $<0,001$ \\
\hline Thiazidiques & $30 / 120(25 \%)$ & $68 / 155(44 \%)$ & 0,001 \\
\hline \multicolumn{4}{|l|}{ Aucune des comorbidités sus-citées } \\
\hline Inhibiteurs calciques & $235 / 530(45 \%)$ & $273 / 518(52 \%)$ & 0,007 \\
\hline Antagoniste de l'angiotensine 2 & $146 / 530(28 \%)$ & $201 / 518(39 \%)$ & $<0,001$ \\
\hline Thiazidiques & $172 / 530(32 \%)$ & $191 / 518(37 \%)$ & 0,13 \\
\hline
\end{tabular}

Table 3. Prescription des inhibiteurs calciques, des antagonistes de récepteurs de l'angiotensine 2 et des diurétiques thiazidiques dans différents sous-groupes. Données exprimées en nombre de patients (pourcentage) et comparées par test du Chi-2.

*Cardiopathie ischémique, accident vasculaire cérébral ou artériopathie oblitérante 
Contrôle de la pression artérielle (PA) sous bithérapie $\mathrm{PA} \leq 140 / 90 \mathrm{mmHg}$ $79 / 259(31 \%)$

$61 / 141(43 \%) \quad 0,01$

PA systolique (mmHg)

$151,74( \pm 23,54)$

$144,02( \pm 21,15)$

0,001

PA diastolique $(\mathrm{mmHg})$

$89,01( \pm 0,78)$

$85,91( \pm 0,98)$

0,02

Contrôle de la pression artérielle sous trithérapie

$\mathrm{PA} \leq 140 / 90 \mathrm{mmHg}$

$37 / 133(28 \%)$

65/191 (34\%)

0,24

PA systolique $(\mathrm{mmHg})$

$155,77( \pm 25,51)$

$149,34( \pm 25,52)$

0,03

PA diastolique $(\mathrm{mmHg})$

$86,50( \pm 1,28)$

$85,52( \pm 1,06)$

0,59

Table 4. Contrôle de la pression artérielle dans les associations médicamenteuses comportant un diurétique thiazidique ou n'en comportant pas. Résultats exprimés en nombre de patients (pourcentage) ou moyenne (土écart-type) et comparés respectivement avec le test du Chi-2 et le test $\mathrm{t}$ de Student. 